\title{
On-Orbit Evaluations of the Three Axis Stabilization Performances of Standard Micro-Satellite Buses
}

\author{
By Kikuko MIYATA ${ }^{1)}$ and Shinichi NAKASUKA ${ }^{2)}$ \\ 1) Department of Aeronautics and Astronautics, Nagoya University, Nagoya, Japan \\ 2) Department of Aeronautics and Astronautics, The University of Tokyo, Tokyo, Japan
}

(Received July 31st, 2015)

\begin{abstract}
In early 2014, three micro-satellites, named UNIFORM-1, HODOYOSHI-3, and HODOYOSHI-4, were launched. These satellites used the newly developed standard micro-satellite bus system, which aims to reduce the development period and cost of a satellite with the help of a recoverable system concept. This paper describes the system concept, design summary, on-ground evaluation system, and on-orbit performance of the attitude determination and control system. The satellites have achieved initial attitude acquisition and shown acceptable levels of three-axis stabilization performance and system survivability despite unexpected anomalies in the components. In its discussion of on-orbit performance, this paper focuses on the three-axis stabilization phase. The unexpected problems are summarized and the procedures for adjusting to the anomalies are explained.
\end{abstract}

Key Words: Micro-satellite, Attitude Determination and Control System, Standard Bus, On-orbit Evaluations

\section{Introduction}

Compared with mid- or large-sized satellites, small satellites have remarkable advantages in terms of development cost and time. To enhance the benefits of small satellites, the University of Tokyo and the Next-generation Space-system Technology Research Association (NESTRA) have developed a novel standard micro-satellite bus system supported by the Japanese government's Funding Program for World-Leading Innovative R\&D on Science and Technology (FIRST). The satellite system is intended to be a straightforward system to achieve good cost effectiveness and a short development period by introducing the concept of a "recoverable system." This system can charge batteries using an automatic "safe mode" transition during most possible anomaly cases. ${ }^{1)}$ The system has been applied to three micro-satellites.

These satellites have achieved their initial attitude acquisition $^{2)}$ and low-accuracy three-axis stabilization. The mission operations are proceeding as reported in 3)-5). This paper focuses on the attitude determination and control system (ADCS) and discusses the on-orbit three-axis stabilization performances of the three satellites.

\section{Satellite System Overview}

To meet the H-IIA piggyback launch constraints, the bus system of the satellite is limited to a volume of less than 50 $\mathrm{cm}^{3}$ and a mass of $50 \mathrm{~kg}$. The system's structure can be extended to improve mission flexibility.

\subsection{Recoverable system}

The satellite system's survivability is related to battery charging. Each satellite operation mode is related with power consumption. This satellite system has a minimum function mode, called the "safe mode," which allows it to survive under anomalies. The function and equipment configuration of the mode are selected to charge the battery in passive operation without attitude stabilization. ${ }^{1), 2)}$ The satellite system changes its operation modes automatically with the help of a battery voltage measurement and moves to the safe mode when the battery voltage is very low. Therefore, the designed satellite bus system can recover from most possible anomaly situations. The recovery sequences are connected to the nominal operation sequence to reduce the effort required for analysis and testing. In case of emergency, the satellite system can reset and restart itself automatically. The operator can also send a reset and restart command from the ground station.

\subsection{Adjustability for multiple mission opportunities}

The satellite has two sets of bus data processors and one computer specialized for mission data. In addition to the satellite bus system, there are mission specialized communication system and power distribution switches. This concept enables adjustability for multiple mission opportunities. The two types of bus data processors support parallel development and reduce the development time. One is the main on-board computer (MOBC) for command, data-handling, and power and time management. The other is the attitude-control on-board computer (AOBC) for attitude control. The functions of these two computers can be installed into a single computer, if needed.

As shown in Fig. 1, the mission system has an independent data processor, transmitter, and power distribution switch, and minimum connection with the bus system. Therefore, the system can easily adjust to different missions with little change in the bus. 


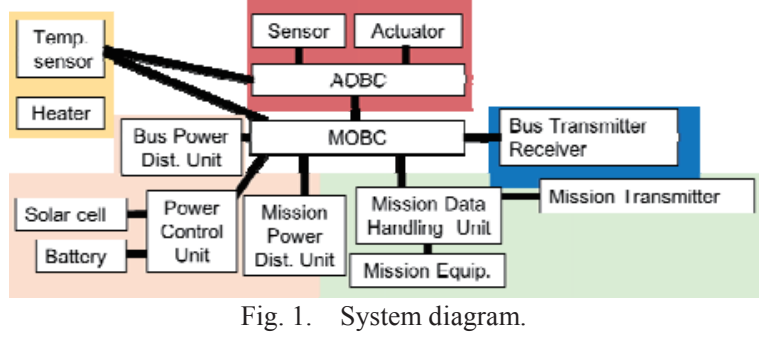

\subsection{Three satellites for an on-orbit case study}

The standard bus system is applied to the three satellites, as shown in Fig. 2.

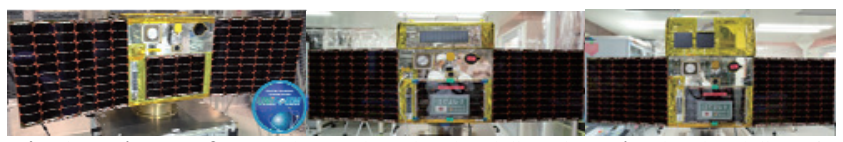

Fig. 2. Photos of UNIFORM-1, HODOYOSHI-3, and HODOYOSHI-4.

All satellites perform Earth remote-sensing missions. The satellites, from left to right, are UNIFORM-1 (launched by H-IIA on 2014/5/24), HODOYOSHI-3, and HODOYOSHI-4 (both launched by Dnepr on 2014/6/20).

\section{Overview of the ADCS}

This section provides an overview of the ADCS. First, the system and mission requirement are summarized. Then, component selection and software design are explained. Finally, the modes and mode transitions are defined.

\subsection{Summary of the requirements}

The satellite system's requirements are as follows:

1) Sun-pointing attitude acquisition after separation from the launcher or recovery from the system's safety mode

2) three-axis Sun-pointing stability as the nominal attitude

3) a special mode for battery charging

4) Earth-centered acquisition attitude for a mission data downlink

The main mission components require three-axis stabilization. The detailed requirements for full mission equipment performance are summarized in Table 1.

Table 1. ADCS requirements for missions.

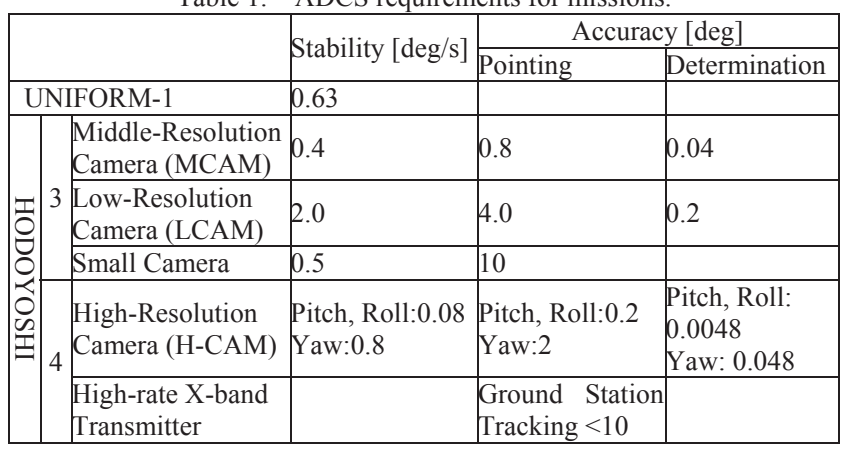

In addition, the following functions are required:

1) Offset-pointing: multiply the offset angle from the target attitude by a commanded value

2) Avoidance of attitude change during operation in imaging missions
3) Avoidance of sunlight incident on the specific mission sensor

\subsection{Component configuration of the ADCS}

The system diagram of the ADCS is shown in Fig. 3. The command and data-handling, time, and power management are performed through the MOBC. The components' power switches are controlled through the bus component power distribution unit (BPDU).

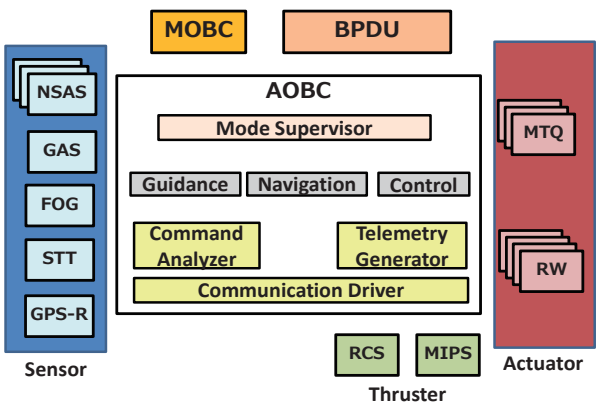

Fig. 3. ADCS diagram.

To fulfill the mission requirement, a precise three-axis attitude control is needed. Therefore, a set of four reaction wheels (RWs) is selected as the main actuator. To avoid zero-crossing, the actuators are mounted with three axes and one skew configuration. ${ }^{6}$ A set of three magnetorquers (MTQs) is selected as the angular momentum controller because of their low power consumption and lack of fuel requirements.

For the state estimation, a geomagnetic aspect sensor (GAS) is mounted because the magnetic control needs geomagnetic field measurements. The precise attitude control requires precise attitude determination; therefore, a star tracker (STT) is selected as the main attitude sensor. Highly accurate body rate data are needed to complement the STT measurements, and a fiber optics gyro (FOG) is selected for this purpose. A GPS receiver (GPS-R) with omnidirectional antennas obtains the precise orbital measurement data. In addition, three non-spin Sun aspect sensors (NSASs) are mounted to ensure the power generation and primitive attitude information. Fig. 4 shows the body axis definition, alignments of the components, and fields of view.

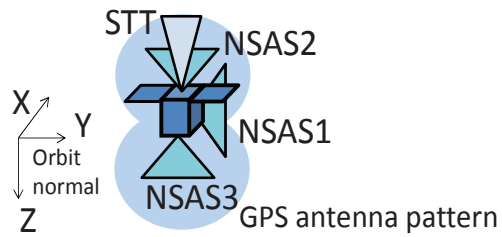

Fig. 4. Sensor's alignment, field of view, and antenna pattern of GPS-R.

Most of the components are newly developed for this program. Overviews are summarized in Table 2. In addition, HODOYOSHI-3 and 4 have propellant systems as mission equipment; HODOYOSHI-3 has a $\mathrm{H}_{2} \mathrm{O}_{2}$ thruster called the reaction control system (RCS), while HODOYOSHI-4 has a miniature ion thruster propulsion system (MIPS). The command- and data-handling of these equipment are performed by the AOBC. 
Table 2. Component specification overview.

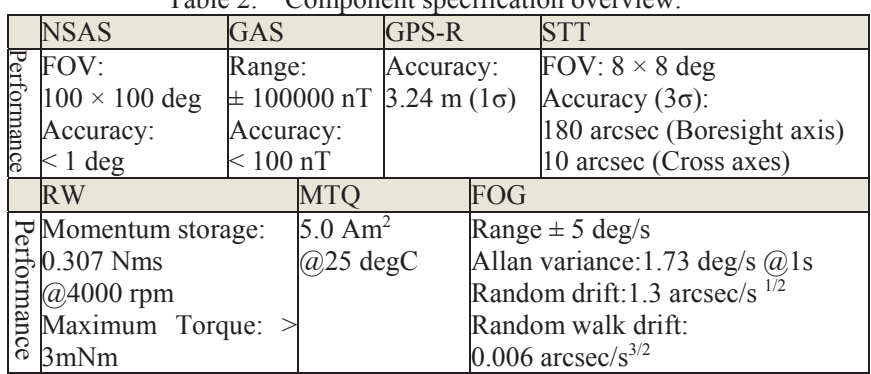

The AOBC software has five main functions: 1) mode supervision, 2) state estimation, 3) target state calculation, 4) required control parameter calculation, and 5) data processing.

In the part responsible for attitude-control logic, the software is designed to add mode reconfiguration and on-orbit performance-improvement ability through parameter tuning.

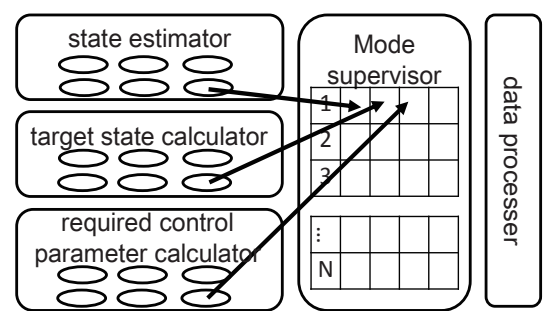

Fig. 5. Overview of mode reconfiguration.

The ADCS mode is mainly defined as the satellite's operation mode. Fig. 5 shows an overview of mode reconfiguration. In the attitude-control logic, each function is divided into module blocks, which are then numbered. The mode sequences are defined in the mode supervisor table, which defines the sequence on the order of the module block. The operator can change the logic sequence and the size of the table by command upload.

Performance-related parameters are also chosen before the flight and coded so as to enable updating by command.

\subsection{Overview of the ADCS mode definition}

Based on the requirements and configuration of the components, there are five main ADCS operation modes: 1) no control, 2) spin Sun acquisition, 3) rough Sun acquisition, 4) rough Earth acquisition, and 5) precise Earth acquisition. Each mode's power consumption and the mode transitions are defined to achieve a recoverable system.

The active components and electric power balance under the expected attitude condition of each mode are summarized in Table 3. The relationship between the attitude mode and mission requirement is summarized in Table 4.

Table 3. Components and power consumption list in each mode.

\begin{tabular}{|c|c|c|c|c|c|c|}
\hline & & No control & Spin Sun & Rough Sun & Rough Earth & Precise Earth \\
\hline \multirow{11}{*}{ 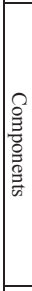 } & MOBC & \begin{tabular}{|c|}
$\circ$ \\
\end{tabular} & 0 & 0 & 0 & 0 \\
\hline & Receiver & $\circ$ & $\circ$ & $\circ$ & $\circ$ & $\circ$ \\
\hline & Power components & $\circ$ & 0 & 0 & $\circ$ & $\circ$ \\
\hline & AOBC & & $\overline{0}$ & $\overline{0}$ & $\overline{0}$ & $\overline{0}$ \\
\hline & GAS & & $\circ$ & $\circ$ & $\circ$ & o \\
\hline & NSAS & & $\circ$ & $\circ$ & $\circ$ & \\
\hline & MTQ $(1,2,3)$ & & $\circ$ & $\circ$ & $\circ$ & 0 \\
\hline & FOG & & $\circ$ & $\circ$ & $\circ$ & $\circ$ \\
\hline & RW-1,2,3,4 & & & 음 & $\circ$ & $\circ$ \\
\hline & STT & & & & & 요 \\
\hline & GPS-R & & & $\overline{0}$ & $\circ$ & $\circ$ \\
\hline & Power balance & + & + & + & - & - \\
\hline
\end{tabular}

Table 4. Relationship between the mission instruments and the requirements for the attitude modes.

\begin{tabular}{|c|c|c|c|}
\hline \multicolumn{3}{|c|}{ UNIFORM-1 } & Rough Sun, Rough Earth, Precise Earth \\
\hline \multirow{8}{*}{ 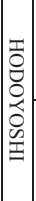 } & & MCAM & Precise Earth \\
\hline & & LCAM & Precise Earth \\
\hline & & Small Camera & Rough Sun, Rough Earth, Precise Earth \\
\hline & & Other mission instruments & Spin Sun, Rough Sun, Rough Earth, Precise Earth \\
\hline & & H-CAM & Precise Earth \\
\hline & & High-rate X-band Transmitter & Rough Sun, Rough Earth, Precise Earth \\
\hline & & MIPS & Rough Earth, Precise Earth \\
\hline & & Other mission instruments & Spin Sun, Rough Sun, Rough Earth, Precise Earth \\
\hline
\end{tabular}

Normally, mode transitions are performed by ground commands; the auto mode transition is only designed to achieve a recoverable system. The trigger is the low battery voltage measurement. The mode transition and power requirements are summarized in Fig. 6.

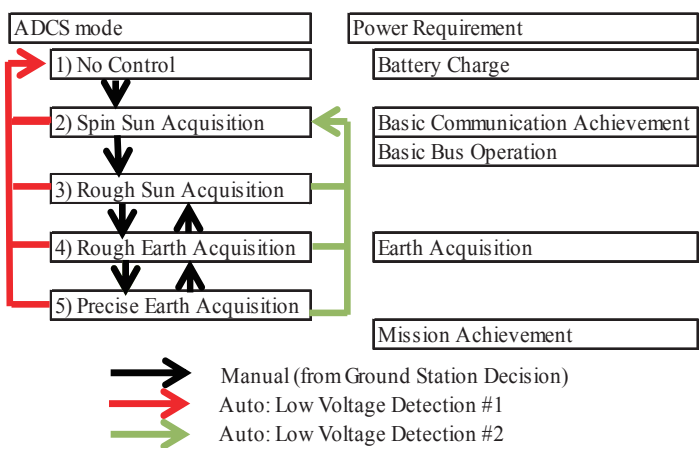

Fig. 6. Summary of the ADCS mode definition and its transition.

\section{Overview of Three-axis Stabilization}

The three-axis stabilization modes are modes 3, 4, and 5 in Fig. 6. In these modes, the system uses RWs as the main actuator and adopts a zero-momentum method. The three-axis control flow considering the requirements is summarized in Fig. 7.

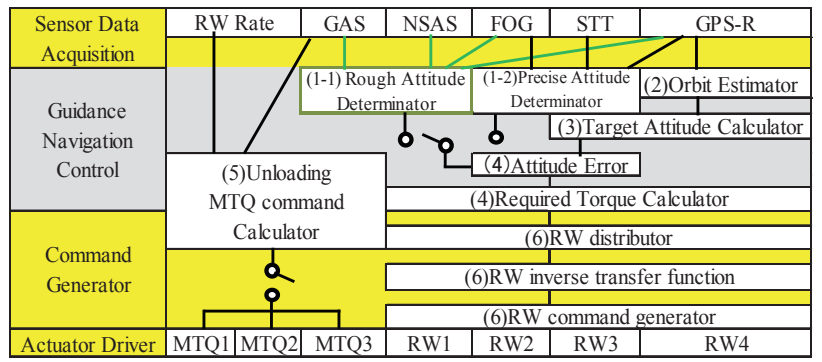

Fig. 7. Summary of three-axis stability flow

This section explains the details of the requirements listed in Fig. 7. First, guidance navigation control is explained through descriptions of the algorithms for state estimation (4.1), target state calculation (4.2), and control requirement calculation (4.3). Other aspects are hardware-related and explained in section 4.4 .

\subsection{State estimator}

The state estimator consists of (1) the satellite attitude determiner and (2) the orbit estimator. There are two types of attitude determiners: One (1-1) is the rough attitude determiner for modes 3 and 4 and the other (1-2) is the precise attitude determiner for mode 5 . 


\subsubsection{Rough attitude determiner}

This method uses NSAS, GAS, GPS-R, and FOG because of their low power consumption. The theoretical Sun direction and geomagnetic vectors are calculated from the Coordinated Universal Time (UTC), satellite position and velocity data. The data are determined from the GPS-R measurements. Comparing these data with the measured Sun direction and geomagnetic vectors, the satellite attitude can be estimated in quaternions in the J2000 coordinate system. The determined attitude is propagated by the FOG measurement with the Kalman filter. ${ }^{6}$

\subsubsection{Precise attitude determiner}

This method uses STT, GPS-R, and FOG. STT gives an attitude quaternion in the J2000 coordinate system. The attitude data are put out at $1 \mathrm{~Hz}$ and are propagated by the FOG measurement with the Kalman filter. ${ }^{6}$

\subsubsection{Orbit estimator}

The satellite position and velocity are calculated in the J2000 coordinate system from the GPS-R measurement. The measurement data are put out at $1 \mathrm{~Hz}$ and propagated by a circular orbit approximation with a Kalman filter.

\subsection{Target state calculator}

In the target state calculation (3), the main target attitude can be selected from the Sun-pointing or the Earth-center-pointing directions. In both cases, the target attitude quaternion is calculated based on the time- and orbit-determination results. As shown in Fig. 8, for the Sun-pointing attitude, the satellite body's Y-axis is normal to the orbital plane, and the $\mathrm{X}$-axis is normal to the $\mathrm{Y}$-axis and the vector from the Earth's center to the Sun. In Earth-pointing, the satellite body $\mathrm{Y}$-axis is normal to the orbital plane, and the Z-axis points to the Earth's center.

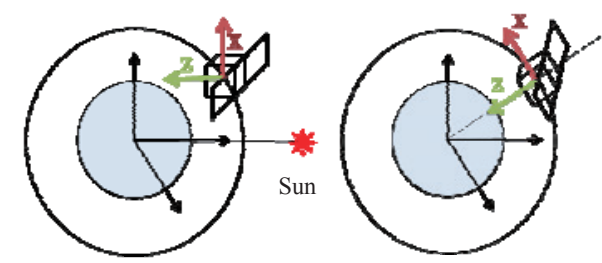

Fig. 8. (left) Sun-pointing and (right) Earth-pointing attitude definition.

The system has additional attitude-pointing modes, one of which is offset-pointing, using which the operator can set the arbitrary offset angle to the target attitude. The other is the absolute target mode, in which the target attitude can be set to an arbitrary inertial attitude from the commanded attitude.

\subsection{Control requirement calculator}

This part consists of the attitude control (4) and the angular momentum control (5).

\subsubsection{Attitude control}

From the determined satellite attitude and the calculated target attitude, the error quaternion is obtained. The required control torque is calculated by the proportional-derivative
(PD) method $^{6}$ from the error quaternion and rate. If the angular errors are small and the satellite system achieves zero momentum, the satellite's equations of motion can be simplified as Eq. (1):

$$
I_{i} \ddot{\theta}_{i}+K_{d i} \dot{\theta}_{i}+K_{p i} \theta_{i}=n_{d i} \quad(\mathrm{i}=1,2,3)
$$

Here, $I_{\mathrm{i}}$ is the moment of inertia, $\theta_{\mathrm{i}}$ is the angular error, $n_{\mathrm{d}}$ is the disturbance torque, $K_{\mathrm{pi}}$ is the proportional gain, and $K_{\mathrm{di}}$ is the derivative gain of each axis. Therefore, the transfer function $G(\mathrm{~s})$ becomes Eq. (2):

$$
\begin{aligned}
G(s) & =\frac{1}{I_{i} s^{2}+K_{d i} s+K_{p i}} \\
& =\frac{1}{I_{i} \omega_{n i}{ }^{2}} \frac{\omega_{n i}{ }^{2}}{s^{2}+2 \zeta_{n i} \omega_{n i} s+\omega_{n i}{ }^{2}}(\mathrm{i}=1,2,3)
\end{aligned}
$$

Then, the gains are defined as

$$
K_{\mathrm{di}}=I_{\mathrm{i}} \omega_{\mathrm{ni}}{ }^{2}, K_{\mathrm{pi}}=2 \zeta_{\mathrm{ni}} I_{\mathrm{i}} \omega_{\mathrm{ni}} .
$$

Here, $\omega_{\text {ni }}$ is the natural frequency and $\zeta_{\text {ni }}$ is the damping ratio. These parameters can be changed from the ground by command. The designed satellite system can use the same " $\omega_{\mathrm{ni}}$ " and " $\zeta$ ni" values for different satellites because the equation includes the moment of inertia term.

\subsubsection{Angular momentum control}

The satellite angular momentum is calculated by the rotation rates of the satellite body and RWs. To maintain the angular momentum under the target value, the satellite generates MTQ operational commands from the calculated angular momentum error, geomagnetic field measurement, and cross-product method. $\left.{ }^{6}\right)$ Some imaging missions require high-attitude stabilization, such as H-CAM. During these mission phases, this control can be removed from the control sequence to achieve high stability.

\subsection{Data processing}

This part includes the hardware specification; the RW-related part (6) is divided into three phases. The calculated required control torque is distributed to fit the RW configuration; if all RWs are under nominal operation, the skew is set to rotate at a constant rate and the other RWs' rotation commands are set to cancel the skew angular momentum in addition to satisfying the torque requirement. If one of the RWs has failed, the required torque is distributed to the other RWs using a pseudoinverse matrix. ${ }^{6}$ ) This part also considers the inverse transfer function and the limit torque of the RWs. In this way, the system generates commands to drive the RWs.

Other commands and data-handling operations related to the components are performed in this layer. To achieve the mentioned algorithms, sensor data are needed. Therefore, the system has a sensor data-acquisition function for all sensors. In addition, there are functions that drive the actuators to generate the required amount of control. 


\section{Ground Evaluation}

This chapter describes on-ground software and hardware verification, especially for the ADCS.

\subsection{Software verification}

The control logic and on-board software are evaluated by a software-based simulator. The evaluation sequence is divided into three phases based on the validation processes shown in Fig. 9. The phases are called (1) model in the loop simulation (MILS), (2) software in the loop simulation (SILS), and (3) hardware in the loop simulation (HILS). The details of each phase are explained below:

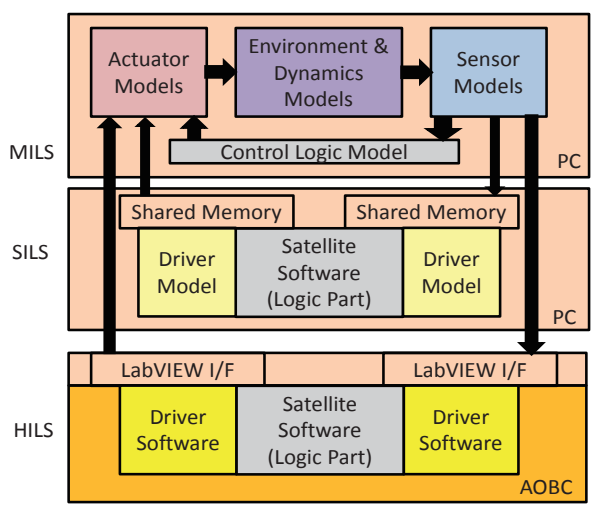

Fig. 9. Overview of the software evaluation environment.

\subsubsection{MILS}

In the first stage, the efficiency of the logic is verified using the full software simulator coded in Matlab. The simulator includes mathematical models of the on-board components and satellite environment, and can perform rapid acceleration experiments.

\subsubsection{SILS}

In this stage, the AOBC logic model is replaced by the real on-board software and the software is tested. The acceleration experiment can be achieved in this phase.

\subsubsection{HILS}

In this stage, the AOBC model is completely replaced by the real hardware to confirm the components' hardware specifications, such as communication delay. This phase can only be performed in real time.

\subsubsection{Mathematical models}

The mathematical models including those of the environment, dynamics, sensors, and actuators are made to be as realistic as possible. Therefore, the sensor and actuator models are updated using the real hardware gathering data, not only during the development phase but also during the on-orbit operation phase. To calculate orbital perturbation, the Earth's gravitational potential (up to J2), solar radiation pressure, and atmospheric drag are modeled. For the attitude disturbance torque, residual magnetism, gravity gradient, atmospheric drag, and solar radiation pressure are modeled. The mechanical parameters and residual magnetism are modeled according to the designed parameters.

\subsubsection{Software-verification sequence}

In the MILS phase, the performance of the designed logic, effects of changes in the parameters, and mode transitions are checked. In SILS, the confirmed logics are coded in the on-board software and evaluated. The results are compared with those of MILS. In the HILS phase, some selected cases are checked and the results are compared with those of SILS. The hardware-related differences, such as communication delays, are also modeled. Then, more realistic parameter tuning is performed in MILS or SILS. In the end, all functions can be confirmed in HILS. Fig. 10 shows the HILS configuration.

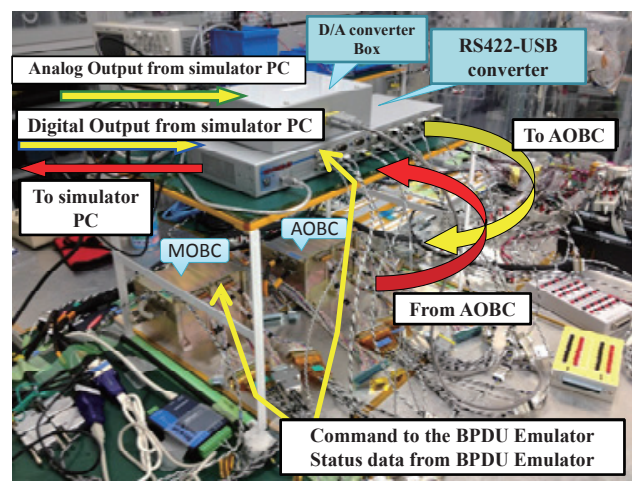

Fig. 10. HILS Environment.

\subsection{Hardware verification}

In addition to software verification, component functional, interface, and environmental testing are also performed. The detailed component performance tests are mainly performed by the vendors. The functional check and environmental tests are mainly performed by the project member. Possible system validations are performed prior to flight.

\section{On-Orbit Results}

All satellites achieved the spin-Sun acquisition at the beginning of their initial operation phases. Then, they moved to the three-axis stabilization mode and achieved a rough Sun acquisition at the beginning of July, 2014. During and after the three-axis stabilization, some anomalies were noticed. The planned operation sequences were changed to endure these anomalies. In this chapter, the initial three-axis operational performances in the rough Sun-acquisition mode are shown. Then, the anomalies are summarized. Finally, overviews of the updated operational plans and some results following updates to the plans are shown.

\subsection{Initial operational results}

This section summarizes the initial operational results, which were obtained in the next visible pass from the main ground station of the mode transition. Figs. 11-13 show the attitude-control error and Figs. 14-16 show the angular rate results. The attitude control error is defined as the difference between the target quaternions and the attitude determination results. The rate results show attitude stability. 


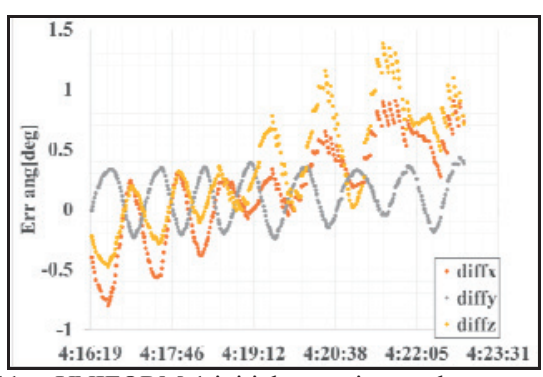

Fig. 11. UNIFORM-1 initial operation angle error results.

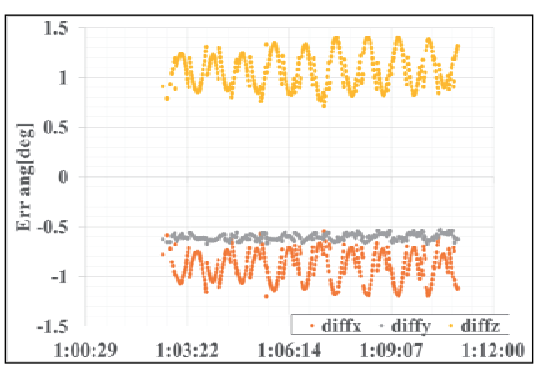

Fig. 12. HODOYOSHI-3 initial operation angle error results.

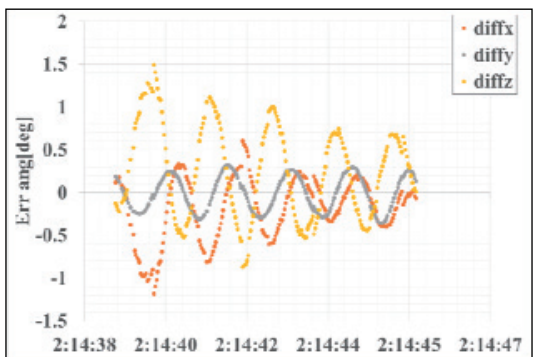

Fig. 13. HODOYOSHI-4 initial operation angle error results.

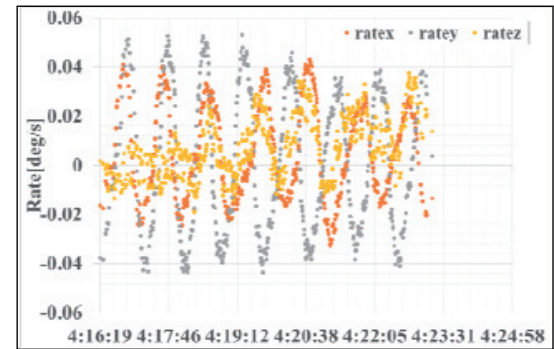

Fig. 14. UNIFORM-1 initial operation angular rate error results.

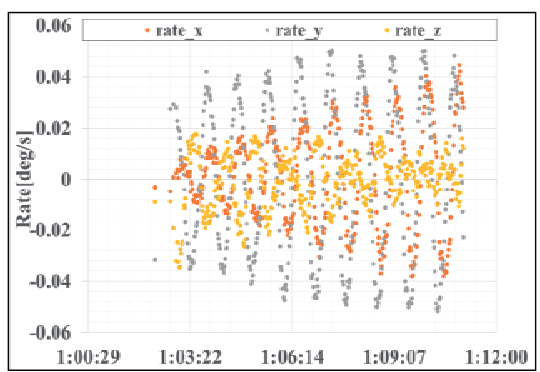

Fig. 15. HODOYOSHI-3 initial operation angular rate error results.

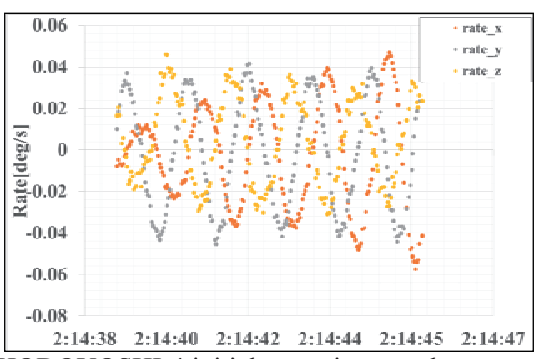

Fig. 16. HODOYOSHI-4 initial operation angular rate error results.
Table 5 shows a summary of the errors. SILS refers to the on-ground SILS simulation results.

Table 5. Summary of $1-\sigma-l e v e l$ angular error and rate stability.

\begin{tabular}{|c|c|c|c|c|c|}
\hline & & \multicolumn{2}{|c|}{ Angular Error [deg] } & \multicolumn{2}{|c|}{ Rate Error $[\mathrm{deg} / \mathrm{s}]$} \\
\hline & $\begin{array}{c}\text { Designed Moments } \\
\text { of Inertia }\left[\mathrm{kgm}^{2}\right]\end{array}$ & SILS & Initial & SILS & Initial \\
\hline UNIFORM-1 & {$\left[\begin{array}{llll}2.11 & 1.78 & 2.19\end{array}\right]$} & 0.25 & 0.56 & 0.025 & 0.036 \\
\hline HODOYOSHI-3 & {$\left[\begin{array}{llll}2.44 & 2.66 & 3.03\end{array}\right]$} & 0.6 & 1.5 & 0.014 & 0.037 \\
\hline HODOYOSHI-4 & {$\left[\begin{array}{llll}2.61 & 3.42 & 3.80\end{array}\right]$} & 0.34 & 0.65 & 0.015 & 0.041 \\
\hline
\end{tabular}

Here, the angular errors are about two times those observed during the simulation and the rate errors are three times their values. Compared with the requirements in Table 1, these rate values show that the system achieved the attitude stabilization requirement, except for H-CAM's pitch and roll requirement. Because these results are initial operation results, the system has not finished angular momentum control. That is why high-attitude stability cannot be achieved in this phase.

To discuss the attitude-pointing accuracy, Figs. 17-19 show the errors in Sun sensors' measurements compared with the absolute target attitude, $|\Delta \theta|$.

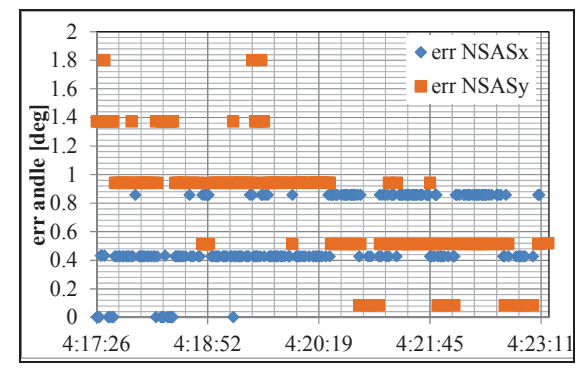

Fig. 17. UNIFORM-1 Sun sensors' error compared with the target attitude.

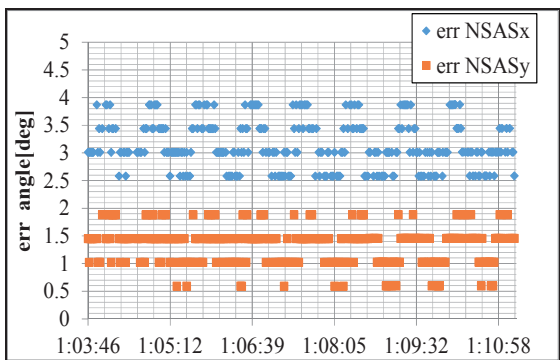

Fig. 18. HODOYOSHI-3 Sun sensors' error compared with the target attitude.

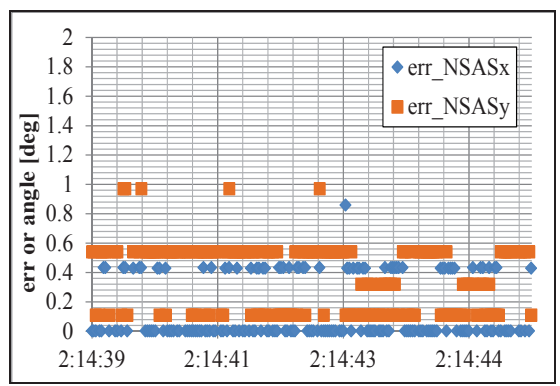

Fig. 19. HODOYOSHI-4 Sun sensors' error compared with the target attitude.

The Sun sensor's accuracy is $1 \mathrm{deg}$. The UNIFORM-1 and HODOYOSHI-4 results show that the pointing error is within 2 deg; however, HODOYOSHI-3's error results are relatively larger than the others. In addition, the differences from their averages show their stabilities and the values are about 1.2 [deg] for HODOYOSHI-3, 0.3 [deg] for HODOYOSHI-4, and 0.4 [deg] for UNIFORM-1. 
HODOYOSHI-3's orbit estimation results might cause the attitude determination and control error. From the hardware constraints, the GPS-R's data acquisition time has some constant error caused by the relationships between the GPS-R serial data output time and the data acquisition board's movement. Therefore, timing problems might occur in HODOYOSHI-3's orbital data acquisition.

\subsection{Anomalies in components}

There were some anomalies in components on-orbit, which were not detected before the flight. Here, these anomalies are summarized.

\subsubsection{NSAS}

NSAS has two main problems. Because of a problem with components' software, anomalous data are output near the edge of the field of view (FOV). Fig. 20 shows a typical case.

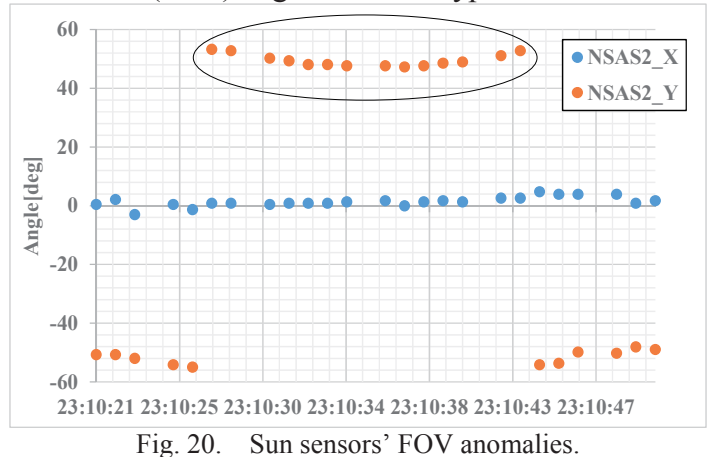

In addition, the sensor detects the Earth's albedo. An example is shown in Fig. 21. Here, the satellite is in the process of acquiring the spin Sun-pointing. The satellite rotates around the Z-axis and the solar paddle faces the Sun's direction. AOBC software cannot detect the anomaly and cannot exclude these abnormal measurements.

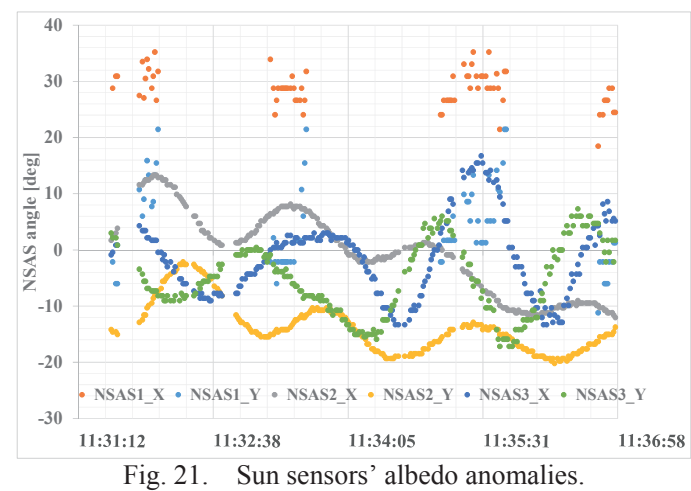

\subsubsection{STT}

The component parameter-tuning problem is found in the internal software. The STT cannot identify the stars and determine attitude. The supplier gave an additional command list to perform on-board parameter tuning, but the debug commands could not be sent because the AOBC software cannot handle undefined commands. Therefore, the precise attitude determiner cannot be used on-orbit.

\subsubsection{Component power control}

There were some failures in the power supply control even with the real-time command. Therefore, the decision was made to use the time-defined power switch control commands only during the visible operation period.

\subsection{On-orbit reconfiguration against component anomalies}

The operational plans are reconfigured to adapt to the anomalies, as mentioned in section 6.2. This section explains some of the operational plans to improve attitude controllability.

\subsubsection{NSASs power control}

After rough Sun-pointing was achieved, only NSAS-2, which faces the Sun, could find that body. Therefore, the power supplies of the other sensors were shut down before the attitude determination to achieve three-axis stability.

\subsubsection{Parameter tuning of the state estimation}

Based on mission requirements, the satellites have to change their attitudes, but this carries risks for the effects of the Sun sensors' anomalies. The system has to ignore the anomalous Sun sensor data, which comes with an albedo or FOV risk. Power switch control is one method for doing so, but the time-defined power switch control command is only used during real-time operation because it has failure risks and has to receive a recover command from the ground station in case of failure.

To avoid the anomalies, Sun sensor data with the possibility of albedo or FOV effects are ignored by parameter tuning. Our software can change the gain matrix of the Kalman filter; by changing this value, the effects of the risky Sun sensor data are cut off and only the healthy Sun sensor data are used for attitude determination. During daily operation, the gain matrices are changed with the time-defined command. The gains are tuned to be smaller only near the equator.

Fig. 22 shows the success of the Sun sensor's anomaly avoidance as an example. During the attitude maneuver, NSAS-2 catches the Sun on the edge of the FOV. However, this does not cause loss of pointing, and the satellite maintains the Sun acquisition after this event.

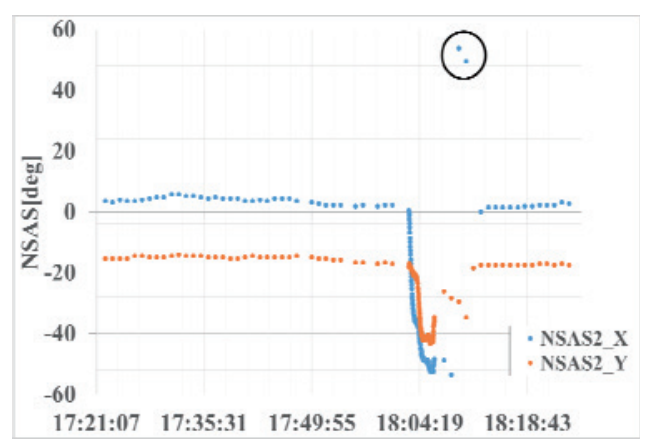

Fig. 22. Attitude stabilization under the anomaly.

\subsubsection{Redefinition of the mode transition}

Because of the Sun sensor and the power switch anomalies, the mode transitions with low-voltage detection are changed. In Fig. 6, the low voltage detection \#2 is canceled because of the risk of the auto-NSAS power control.

\subsubsection{Reconfiguration of the ADCS mode by changing the software module combination}

For smooth mode transitions or improvements in the attitude performance, some additional attitude modes are defined after the launch. The attitude modes can work smoothly on-orbit. 


\subsubsection{Operational plan limitation}

Because of a difference in the software version, the UNIFORM-1 satellite cannot change its gain matrix. Therefore, there are operational limits in the attitude changes to maintain the Sun sensor measurement in the normal range.

\subsubsection{Mission achievement}

All mission equipment obtained some results because the system achieved the stability requirement; however, some equipment had difficulties in obtaining the exact data required because the system could not achieve the pointing and determination requirements. Here, some examples of the mission achievement are shown. Fig. 23 is the total lunar eclipse observation from UNIFORM-1.7) Fig. 24 is a photograph taken by HCAM. ${ }^{8)}$ In addition, Fig. 25 shows the attitude rate results during the mission operation in Fig. 24, as well as the acceptable attitude stability for the HCAM mission.

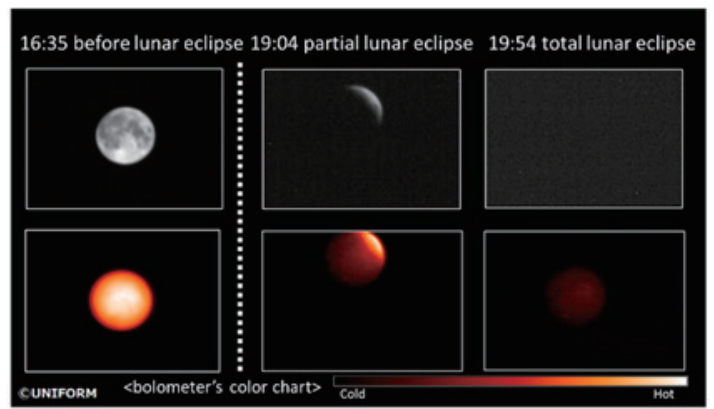

Fig. 23. Total lunar eclipse observation from UNIFORM-1 (October 8, 2014).

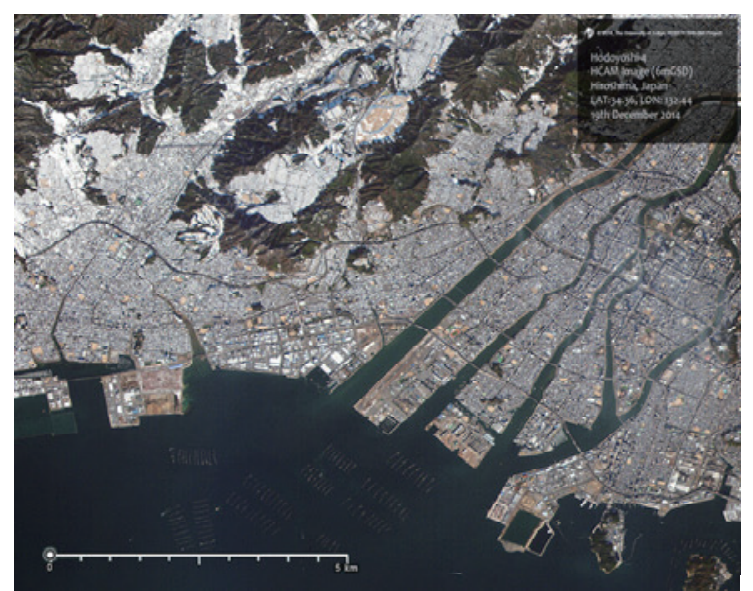

Fig. 24. HCAM image in Hiroshima city, Japan (December 19, 2014).

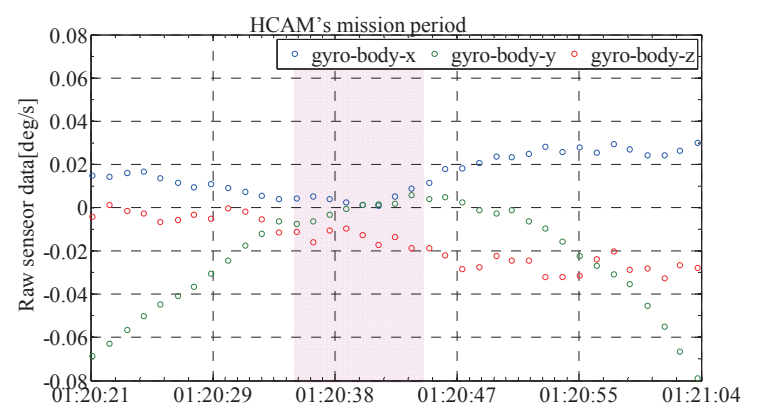

Fig. 25. Angular rate results during the mission operation in Fig. 24.

\section{Conclusion}

The system showed an acceptable level of attitude-control performance, despite anomalies in some of the components. Because of the STT anomalies, the highest level of attitude control could not be achieved. However, the system showed survivability with the help of the "safe mode" transition and updated operational plan. The system recovered from NSAS's anomalies within the designed framework. This means that the design of the "recoverable system" works during the operation. The launched satellites remain under mission operation and continue to provide results.

\section{Acknowledgments}

UNIFORM project is sponsored by the Ministry of Education, Culture, Sports, Science \& Technology in Japan (MEXT) and HODOYOSHI project is granted by the Japan Society for the Promotion of Science (JSPS) through the FIRST Program, initiated by the Council for Science and Technology Policy (CSTP). The authors would like to thank the projects' development and operation teams for their daily effort to progress these projects.

\section{References}

1) Miyata, K. and Nakasuka, S.: ADCS Design with Effective FDIR Considering Mission Constraints for a Small Satellite, Trans. JSASS Aerospace Tech. Japan, 12, ists29 (2014), pp. Td_7-Td_13.

2) Miyata, K. and Nakasuka, S.: Comparison and On-orbit Evaluation of the Initial Attitude Acquisition of Standard Small Satellites, Proceedings of the Symposium on Space Sciences and Technology 58, 6p, 2014-11-12 (in Japanese).

3) Fukami, T., Watanabe, H. et al.: On-Orbit Demonstration of the X-band $348 \mathrm{Mbps}$ Communication System for the Nano Satellite : HODOYOSHI-4, IEICE technical report, 114 (448), 25-30, 2015-02-18.

4) Aoyanagi, Y., Iwasaki, A. et al.: Initial Result of HODOYOSHI-3, 4 Earth Observation Mission, Proceedings of the Symposium on Space Sciences and Technology, 58, 3p, 2014-11-12 (in Japanese).

5) Tanaka, T., Nakasuka, S. et al.: Result of Hosted-Payload Mission of HODOYOSHI3\&4, Proceedings of the Symposium on Space Sciences and Technology, 58, 3p, 2014-11-12 (in Japanese).

6) Attitude Control Research Committee: Spacecraft Dynamics and Control Handbook, Baifukan, Japan, (2007), pp. 450-457, 465-479, 677-684, 724-727 (in Japanese).

7) Institute for Education on Space (IfES), Wakayama University, UNIFORM-1 information page, http://www.wakayama-u.ac.jp/ ifes/UNIFORM/progress/uni1report/uni1-20141008.html (in Japanese).

8) Press release, School of Engineering, The University of Tokyo, http://www.t.u-tokyo.ac.jp/etpage/release/2014/141226_4.html (December 26, 2014). 